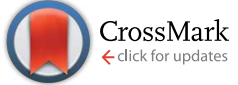

Cite this: RSC Adv., 2017, 7, 7843

\title{
High performance asymmetric supercapacitors with ultrahigh energy density based on hierarchical carbon nanotubes a NiO core-shell nanosheets and defect-introduced graphene sheets with hole structure
}

\begin{abstract}
Zenghui Qiu, Dawei He, Yongsheng Wang, ${ }^{*}$ Xuan Zhao, Wen Zhao and Honglu Wu
In this work, we report a fast and cost-effective cobalt catalyzed gasification strategy to obtain defectintroduced graphene sheets (DGNs) with hole structure. Compared with the pristine graphene, the porous DGNs display much more outstanding capacitive behaviors. An electrode based on the DGNs shows an ultrahigh specific capacitance of $256 \mathrm{~F} \mathrm{~g}^{-1}$ at $1 \mathrm{~A} \mathrm{~g}^{-1}$ and $148 \mathrm{~F} \mathrm{~g}^{-1}$ even at $20 \mathrm{~A} \mathrm{~g}^{-1}$. In addition, hierarchical carbon nanotubes@ $\mathrm{NiO}(\mathrm{CNT}(\mathrm{NNiO})$ core-shell hybrids were fabricated via a facile chemical bath deposition method, followed by thermal annealing. The resulting CNT@NiO electrode shows a considerable specific capacitance of $1000 \mathrm{~F} \mathrm{~g}^{-1}$ at $1 \mathrm{~A} \mathrm{~g}^{-1}$. For the first time, an advanced asymmetric supercapacitor (ASC) device was successfully fabricated consisting of a porous DGN-based negative electrode and a hierarchical CNT@NiO core-shell nanosheet-based positive electrode. The device exhibits a high specific capacitance of $108 \mathrm{~F} \mathrm{~g}^{-1}$ at $0.5 \mathrm{~A} \mathrm{~g}^{-1}$ and an excellent cycling stability, with $93.5 \%$ capacitance retention after 10000 cycles at $6 \mathrm{~A} \mathrm{~g}^{-1}$. Due to its unique microstructures, the CNT@NiO//DGNs ASC device displays a supreme energy density of $38.1 \mathrm{~W} \mathrm{~h} \mathrm{~kg}^{-1}$ at a power density of $500 \mathrm{~W} \mathrm{~kg}^{-1}$ and even retains an energy density of $16.2 \mathrm{~W} \mathrm{~h} \mathrm{~kg}^{-1}$ at $16000 \mathrm{~W} \mathrm{~kg}^{-1}$ (voltage window of $1.6 \mathrm{~V})$. These results indicate that our ASC device is extremely valuable for energy storage applications and predict future trends toward the realization of graphene-based materials used in supercapacitors.
\end{abstract}

Received 26th November 2016 Accepted 8th January 2017

DOI: $10.1039 / c 6 r a 27369 f$

www.rsc.org/advances where their ultrahigh power density (up to $10 \mathrm{~kW} \mathrm{~kg}^{-1}$ ) and ultralong cycle-life $\left(>10^{5}\right.$ cycles) is satisfactory. Supercapacitors have been considered as the most promising power source in newgeneration energy storage systems.

However, because of their limited operating working potential ( 0.6 to $1 \mathrm{~V})$, current symmetrical supercapacitors usually suffer from a lower energy density than rechargeable batteries, which cannot meet the requirements of many practical applications. ${ }^{11-13}$ Therefore, extensive research efforts have been employed to obtain supercapacitors with wider operating voltages and higher energy ${ }^{14-16}$ In terms of the equation:

$$
E=0.5 C V^{2}
$$
double layer capacitors (EDLCs) based on porous carbon materials and pseudocapacitors based on transition metal oxides/ hydroxides. Supercapacitors fill the gap between batteries and conventional dielectric capacitors, ${ }^{6-8}$ and they are extensively utilized in electric vehicles, memory back-up systems, military applications, electronics, and mobile communications etc., ${ }^{8-10}$

Key Laboratory of Luminescence and Optical Information, Ministry of Education, Institute of Optoelectronic Technology, Beijing Jiaotong University, No.3 Shang Yuan Cun, Hai Dian District, Beijing 100044, P. R. China. E-mail:2660779034@qq.com; Fax: +861051688018 we can improve the energy density $(E)$ by maximizing the specific capacitance $(C)$ and/or the operating potential window $(V)$. Asymmetric supercapacitors (ASCs) in aqueous electrolyte are a promising alternative to increase the energy density. ASCs couple a faradaic redox electrode (as the energy source) and a capacitor-type electrode (as the power source) in one system, and can simultaneously offer the advantages of both supercapacitors (rate capability, cycle life) and advanced batteries (energy density), thus making full use of the different potential 
windows of the two electrodes to provide a maximum operation voltage for the whole system, resulting in a large energy density. ${ }^{17,18}$ Therefore, selecting appropriate materials for the positive and negative electrodes of high performance asymmetric supercapacitors has become a major challenge towards their practical application.

Currently, many pseudocapacitive materials such as transition metal oxides/hydroxides have been widely studied as anode materials for ASCs, owing to their high pseudocapacitance and energy densities. ${ }^{19-22}$ Among these metal oxides, NiO is one of the most promising positive pole materials in ASC applications because of its high theoretical specific capacitance $\left(3750 \mathrm{~F} \mathrm{~g}^{-1}\right.$ within $0.5 \mathrm{~V}$ ), low cost, structural stability and easy synthesis. ${ }^{23-25}$ Additionally, NiO-based composites are widely used in some other fields, such as Li-ion batteries and supercapacitors, etc. ${ }^{26-29}$ Nonetheless, like other transition metal oxides, the poor electronic conductivity and low cycle life of $\mathrm{NiO}$ reduce its power performance. An effective way to address this issue is to incorporate nano-sized NiO into electrically conductive frameworks, such as carbon-based materials, to form a NiO-conductive framework core-shell structure. ${ }^{30-33}$ Among these frameworks, CNTs are considered as the most attractive candidate owing to their large surface area, excellent conductivity, good cycle stability and high mechanical strength. ${ }^{34-36}$ Based on this analysis, CNTsupported NiO hybrids can be used as the anode; however, it is still difficult to directly grow ultrathin and quality NiO nanosheets on CNTs via a cost-effective method.

Graphene-based materials have become the most promising cathode materials for asymmetric supercapacitors due to their large surface area and high electrical conductivity. ${ }^{37-40}$ However, graphene-based materials usually suffer from limited specific capacitance, leading to relatively low energy density in supercapacitors. Utilizing graphene-based materials with optimized pore structure has been proven to be a good approach for improving capacitive performance to obtain ASCs with high energy density. ${ }^{41-43}$ To address this issue, the synthesis of defectintroduced graphene sheets (DGNs) with hole structure has attracted increasing attention because of their facile preparation procedures, large surface area, and unique porous structures. Most of the patterning strategies employed, such as electro plasma etching, post-activation processes and lithography, often involve high temperature processing and dangerous gases, which are not cost efficient or suitable for large scale preparation. ${ }^{\mathbf{4 4 - 4 7}}$ Carbon gasification, catalyzed by metallic and oxide particles in a controlled atmosphere, has been demonstrated to be a facile and efficient method. ${ }^{48}$ Based on their highly uniform porosity compared to many other porous carbon materials, DGNs exhibit enhanced electrochemical performance and high-rate capability in asymmetric supercapacitor applications.

Herein, we report a facile and cost-effective cobalt catalyzed gasification strategy to prepare defect-introduced graphene sheets with hole structure. The prepared DGN electrode displays a significantly enhanced specific capacitance $\left(256 \mathrm{~F} \mathrm{~g}^{-1}\right.$ at $1 \mathrm{~A} \mathrm{~g}^{-1}$ ), which is higher than most of the previously reported carbon-based material electrodes. The preparation of hierarchical carbon nanotubes@NiO core-shell hybrids through a chemical bath deposition process with thermal annealing is also reported. Furthermore, our optimized ASC device comprising hierarchical carbon nanotubes@NiO core-shell nanosheets as the anode and defect-introduced graphene sheets with hole structure as the cathode was successfully fabricated for the first time. The device exhibits remarkable electrochemical performance with a maximum energy density of $38.1 \mathrm{~W} \mathrm{~h} \mathrm{~kg}^{-1}$ at a power density of $500 \mathrm{~W} \mathrm{~kg}^{-1}$ and good cycling stability ( $94 \%$ capacitance retention after 10000 cycles) at a wide operational voltage of $1.6 \mathrm{~V}$. These results indicate that transition metal oxides/hydroxides and porous graphene are promising candidates for next-generation energy-storage devices with excellent electrochemical properties.

\section{Experimental section}

\subsection{Synthesis of hierarchical carbon nanotubes@NiO core- shell nanosheets}

To make CNTs more dispersible in deionized water, $1.5 \mathrm{~g}$ of CNTs was refluxed in $\mathrm{HNO}_{3}(6 \mathrm{M}, 100 \mathrm{~mL})$ at $85^{\circ} \mathrm{C}$ for $3 \mathrm{~h}$, followed by washing with copious amounts of deionized water and ethanol. Then the precursor CNTs were dried at $60{ }^{\circ} \mathrm{C}$ overnight for further use. Hierarchical CNT@NiO core-shell nanosheets were then synthesized using chemical bath deposition combined with thermal annealing. Typically, $0.5 \mathrm{~g}$ of the treated CNTs were added into a $200 \mathrm{~mL}$ solution containing $14.5 \mathrm{~g}$ of $\mathrm{NiO}\left(\mathrm{NO}_{3}\right)_{2} \cdot 6 \mathrm{H}_{2} \mathrm{O}$ and $60 \mathrm{~g}$ of urea and the solution was ultra-sonically agitated to form a homogeneous suspension. The mixture was then heated in an oil bath at $90{ }^{\circ} \mathrm{C}$ for $3 \mathrm{~h}$ with stirring, and then the precipitate was cooled down to room temperature and aged for another $12 \mathrm{~h}$ naturally. The initial product was collected via filtration and washed with ethanol and deionized water several times with the assistance of a centrifuge, and was then dried in air at $60{ }^{\circ} \mathrm{C}$ for 12 h. Finally, the precursors were converted into CNT@NiO composites via thermal annealing at $350{ }^{\circ} \mathrm{C}$ for $2 \mathrm{~h}$ with a slow heating rate of $1^{\circ} \mathrm{C} \mathrm{min}^{-1}$. For comparison, the bare $\mathrm{NiO}$ powder was also prepared using the same procedure as described above without CNTs.

\subsection{Preparation of defect-introduced graphene sheets (DGNs) with hole structure}

Graphene was manufactured via the hydrothermal reduction of graphene oxide (GO) in the presence of L-ascorbic acid. The oxidation of graphite powder (GP) to GO was achieved through a modified Hummers' method. In a typical process, $50 \mathrm{mg}$ of asprepared GO and $100 \mathrm{mg}$ of L-ascorbic acid were diffused in 40 $\mathrm{mL}$ deionized water with ultra-sonic agitation for $40 \mathrm{~min}$. The mixture was transferred into a $50 \mathrm{~mL}$ Teflon autoclave and heated in a muffle furnace at $180{ }^{\circ} \mathrm{C}$ for $8 \mathrm{~h}$. The product was washed with deionized water and ethanol, and then dried using lyophilization in order to obtain non-agglomerated graphene powder. The processed graphene sheets (GNs) were impregnated with $10 \mathrm{wt} \%$ Co by dispersion in ethanol and blending fully with stirring. Then, the as-prepared Co/GNs underwent oxidation in air at $300{ }^{\circ} \mathrm{C}$ for $25 \mathrm{~min}$. To obtain the pure DGNs, cobalt was removed in a boiling concentrated nitric acid solution for 50 minutes. Finally, the resulting precipitate was 
filtered off, soaked in ethanol and deionized water several times and then dried with lyophilization. Based on the conclusion that the size and density of hole defects are related to the size and mass of Co catalysts, preventing the agglomeration of $\mathrm{CoO}_{x}$ particles and controlling the mass of Co catalysts are key in the formation of hole structures.

\subsection{Characterization methods}

The structure and morphologies of the samples were characterized using field emission scanning electron microscopy (FESEM, HITACHI S-4800) and transmission electron microscopy (TEM, JEOL JEM-2010F). High-resolution transmission electron microscopy (HRTEM), energy dispersive X-ray spectroscopy (EDS) mapping and selected area electron diffraction (SAED) patterns were measured on a JEOL 2010F microscope. Powder X-ray diffraction (XRD) patterns were recorded on a BRUKER (Germany) D8 advance diffractometer with $\mathrm{Cu}-\mathrm{K} \alpha$ radiation $(\lambda=0.15406 \mathrm{~nm})$. Raman spectra were obtained under ambient conditions using a Renishaw Invia micro-Raman system with an Ar-ion laser beam of $532 \mathrm{~nm}$. Thermogravimetric analysis (TGA) with a thermogravimetric analyzer was conducted under an air atmosphere at a heating rate of $10{ }^{\circ} \mathrm{C}$ $\min ^{-1}$ to characterize the quality change. The BrunauerEmmett-Teller (BET) specific surface area and $\mathrm{N}_{2}$ adsorptiondesorption isotherms of the samples were measured at $77 \mathrm{~K}$ using an automatic adsorption instrument (LabRAM, HREvolution), and the pore size distribution curve was calculated using the Barrett-Joyner-Halenda $(\mathrm{BJH})$ method from the adsorption branch isotherms.

\subsection{Electrochemical measurements}

Working electrodes were prepared by mixing the active materials, carbon black and polytetrafluoroethylene (PTFE), with ethanol in a mass ratio of $80: 10: 10$ to obtain a homogeneous slurry. Then the slurry was pressed (under a pressure of $10 \mathrm{MPa}$ ) onto the nickel foam current collector $\left(1 \times 1 \mathrm{~cm}^{2}\right)$ and dried at $100{ }^{\circ} \mathrm{C}$ for $12 \mathrm{~h}$ in a vacuum oven. The masses of active material on the CNT@NiO electrode, NiO electrode, CNT electrode, DGN electrode, and GN electrode were about $0.75 \mathrm{mg}, 0.75 \mathrm{mg}, 1 \mathrm{mg}, 1.5 \mathrm{mg}$ and $1.5 \mathrm{mg}$, respectively. The electrochemical performances of the individual electrodes were investigated under a three-electrode system, in which platinum foil and a saturated calomel electrode (SCE) were used as the counter and reference electrodes, respectively. During the testing of the optimized asymmetric supercapacitor, the loading mass ratio of the anode material and cathode material (CNT@NiO : DGNs) was estimated to be 0.5, according to their cyclic voltammetry curves and galvanostatic charge-discharge curves (CNT@NiO electrode within 0-0.5 V and DGN electrode within -1-0 V). The CNT@NiO anode and DGN cathode were pressed together and separated by a porous non-woven cloth
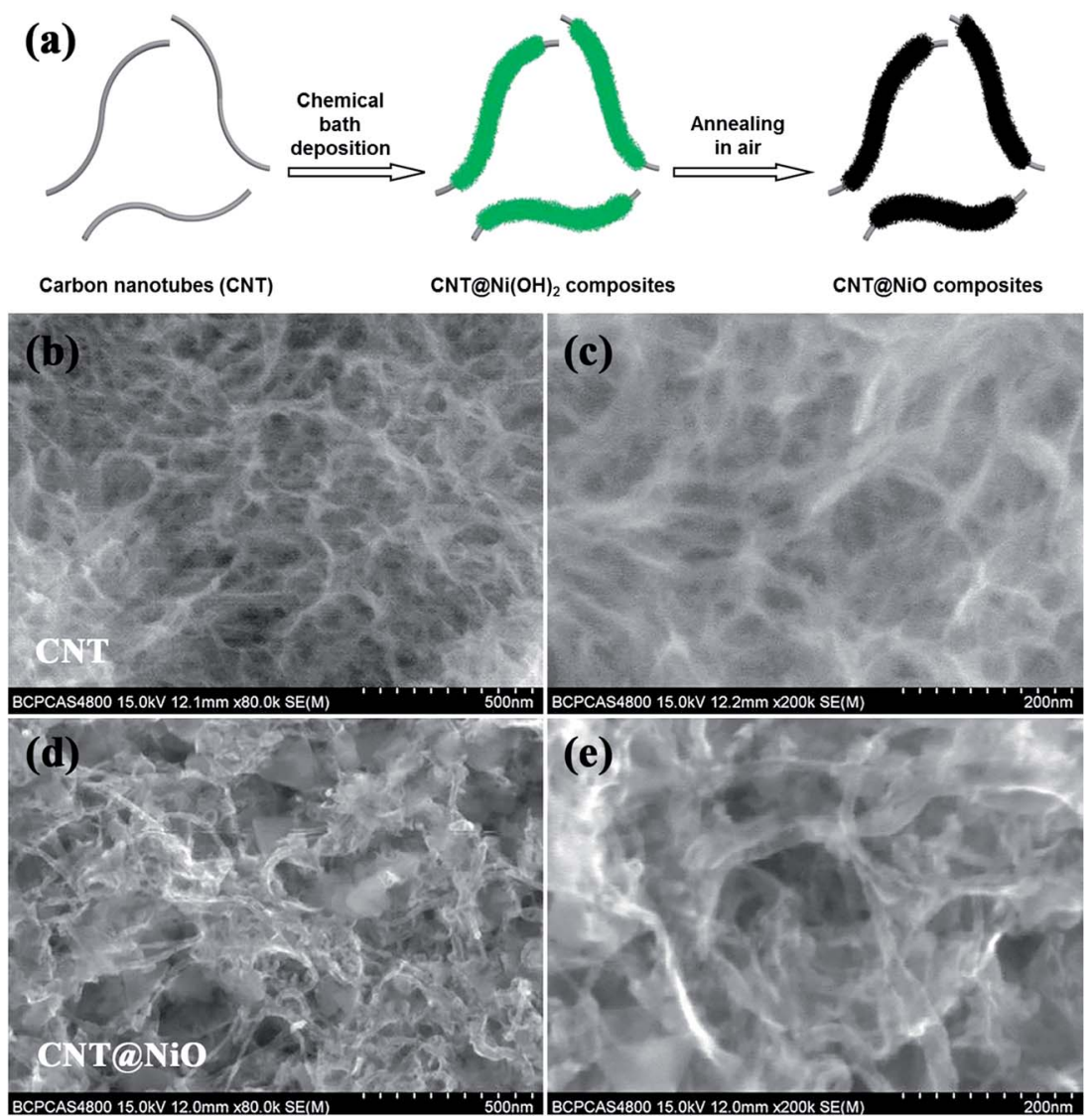

Fig. 1 (a) Schematic illustration detailing the preparation of hierarchical CNT@NiO core-shell nanosheets. FESEM images of (b and c) CNT and (d and e) CNT@NiO core-shell composites. 
separator and electrochemical tests on the asymmetric supercapacitor were performed using a two-electrode cell. All of the above electrochemical measurements were obtained at room temperature in freshly prepared $1 \mathrm{M} \mathrm{KOH}$ aqueous electrolyte using a CHI 660B electrochemical workstation.

\section{Results and discussion}

\subsection{Fabrication and characterization of hierarchical CNT@NiO core-shell electrodes}

The detailed preparation procedure for the hierarchical CNT@NiO core-shell nanosheets is illustrated schematically in
Fig. 1a. Firstly, ultrathin $\alpha-\mathrm{Ni}(\mathrm{OH})_{2}$ nanosheets were grown vertically onto CNTs via chemical bath deposition. Secondly, the CNTs covered with ultrathin $\alpha-\mathrm{Ni}(\mathrm{OH})_{2}$ were annealed to obtain CNT@NiO core-shell nanosheets under an air atmosphere. The morphology and structure of the samples were characterized using FESEM. Fig. 1b and c display the FESEM images of the CNTs under the different magnifications and the CNTs exhibit nanowire structures with a smooth surface and an outer diameter of about 20-60 nm. For the CNT@NiO core-shell hybrids, the NiO nanosheets were uniformly anchored on the surface of the individual CNT in Fig. 1d and e, and their precursor CNT@Ni $(\mathrm{OH})_{2}$ composites provide the core-shell
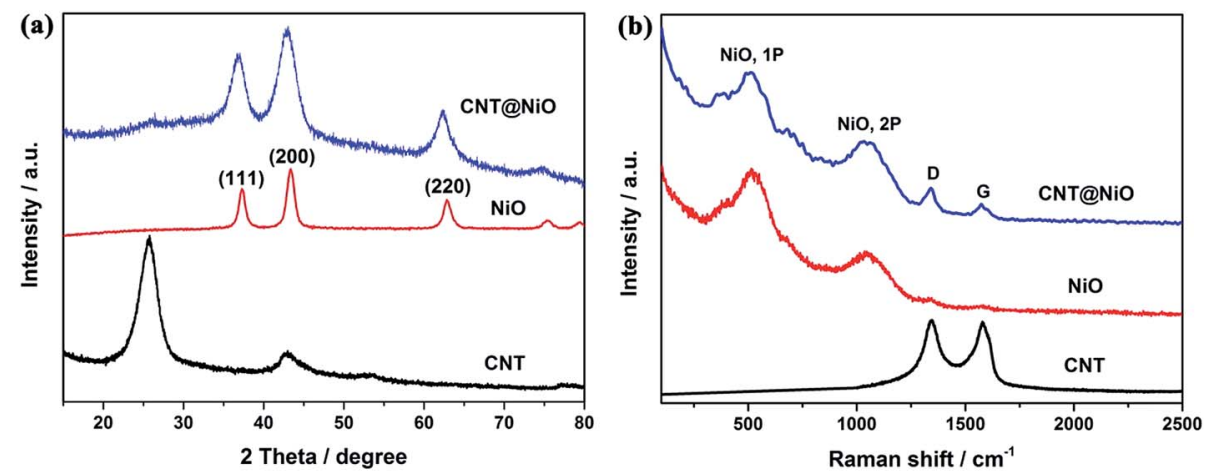

Fig. 2 (a) XRD patterns and (b) Raman spectra of CNT, pure NiO and CNT@NiO core-shell compounds.
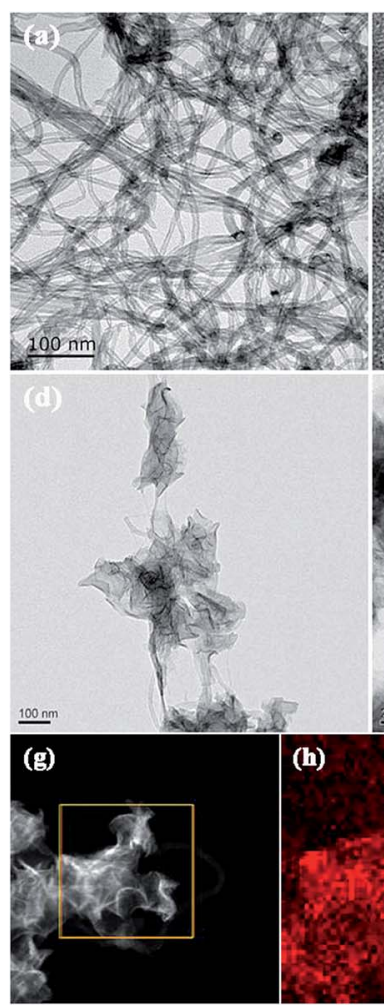

\section{(6).}

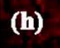

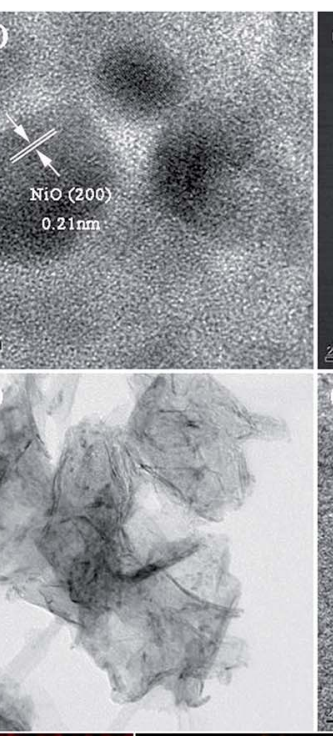

(i) (c)
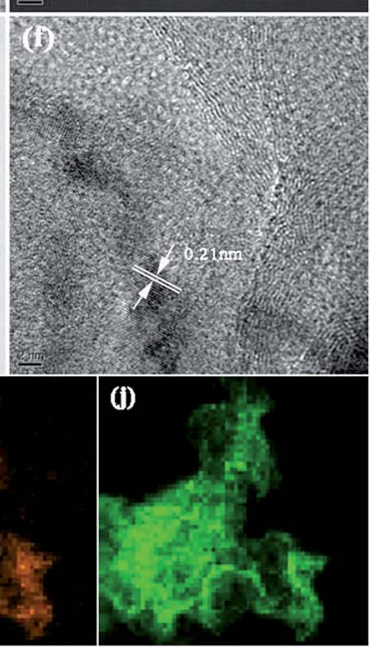

Fig. 3 Typical TEM images of (a) CNT and ( $d$ and e) CNTaNiO core-shell nanosheets. HRTEM images of (b) NiO and (f) CNT@NiO core-shell composites. (c) The SAED pattern of $\mathrm{NiO}$. (g) EDS image and corresponding elemental mapping images of (h) $\mathrm{C}$, (i) $\mathrm{O}$ and (j) $\mathrm{Ni}$ on a single CNT@NiO core-shell nanosheet. 
nanostructure. The interconnections between the NiO nanosheets provide remarkable mechanical strength and form a desirable conductive network not easily separated from the CNT core. Furthermore, the interconnected NiO nanosheets exhibit a highly porous reticulation, which can offer a higher surface area and more active sites related to electrolyte ions. Due to the fact that polymerized micro-sized particles are only present in the bare NiO sample, we can conclude that the CNT shell not only serves as the conductive support for the growth of $\mathrm{NiO}$ core, but also prevents the polymerization of $\mathrm{NiO}$.

The crystallographic structures and phase information for the samples were investigated using typical XRD measurements. As shown in Fig. 2a, the XRD patterns of the pure NiO and CNT@NiO hybrids show three diffraction peaks located at $2 \theta=37.2^{\circ}, 43.2^{\circ}$ and $62.8^{\circ}$, identified as the (111), (200) and (220) planes, respectively, which can be indexed to centered-
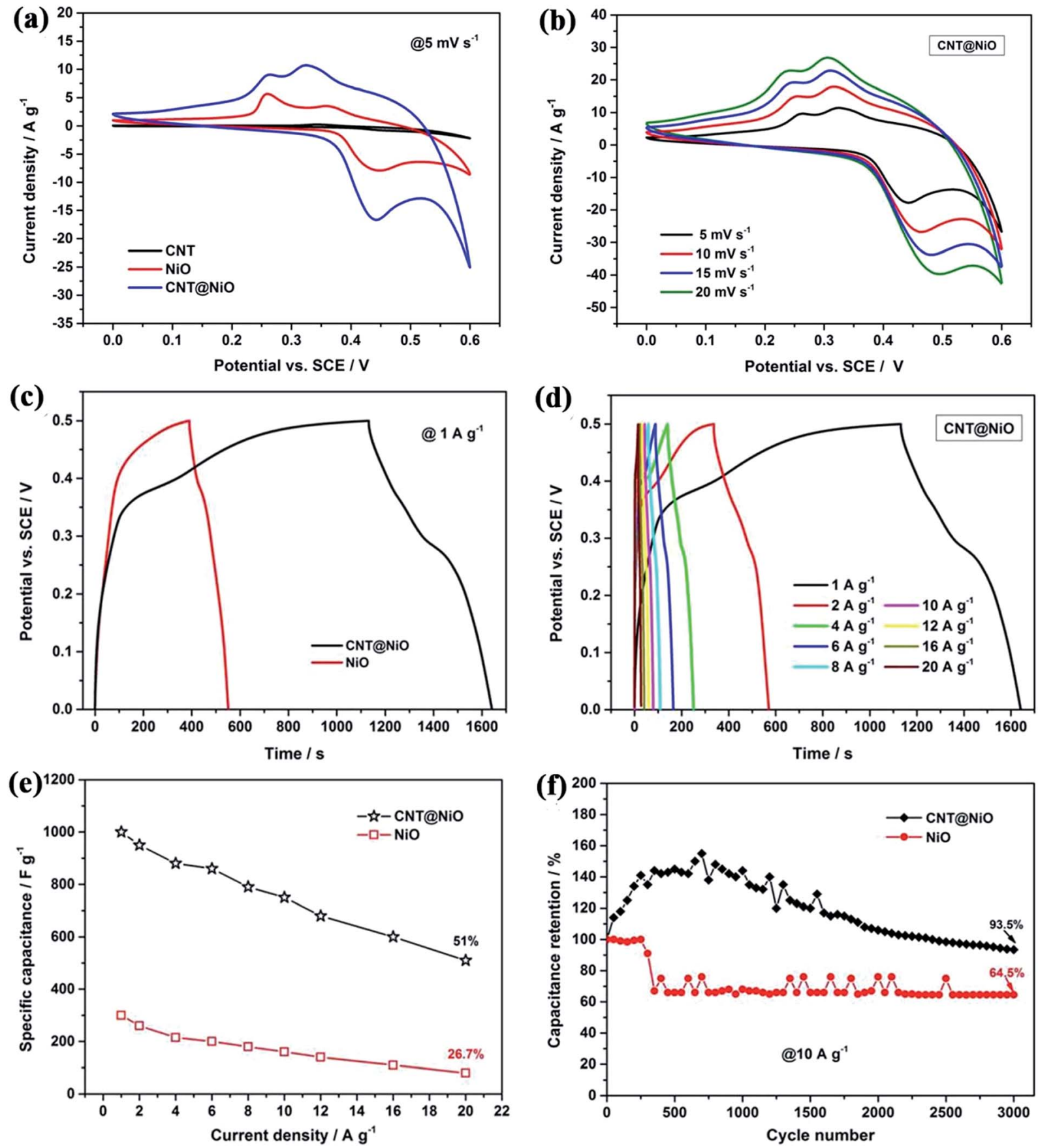

Fig. 4 (a) Comparison of CV curves of CNT, pure NiO and CNTaNiO nanosheets at a scan rate of $5 \mathrm{mV} \mathrm{s}^{-1}$ and (b) CNT (aNiO hybrids at different scan rates. (c) Comparison of GCD curves of pure $\mathrm{NiO}$ and CNTaNiO composites at $1 \mathrm{~A} \mathrm{~g} \mathrm{~g}^{-1}$ and (d) CNT (aNiO hybrids at different current densities. (e) Specific capacitance of pure $\mathrm{NiO}$ and $\mathrm{CNTQNiO}$ compounds at various current densities. (f) Cycling performance of pure NiO and CNTaNiO hybrids at a current density of $10 \mathrm{~A} \mathrm{~g}^{-1}$. 
cubic NiO (JCPDS 43-1477). Obviously, the diffraction graphitic peak at around $26^{\circ}$ is also observed in the CNT@NiO composites. $^{49}$ In addition, compared to bare CNTs, the peaks of the hybrids show weaker diffraction intensity, implying that the $\mathrm{NiO}$ nanosheets were successfully decorated onto the CNTs. Furthermore, no visible XRD peaks of $\alpha-\mathrm{Ni}(\mathrm{OH})_{2}$ are observed in the CNT@NiO sample, affirming the complete conversion of $\mathrm{Ni}-\mathrm{OH}$ into Ni-O during the annealing process. The samples of CNTs, pure NiO and hierarchical CNT@NiO core-shell nanosheets are further confirmed by the Raman spectra shown in Fig. 2b, which display three peaks at about $1343 \mathrm{~cm}^{-1}$ (D-band) and $1577 \mathrm{~cm}^{-1}$ (G-band), along with a peak at $2691 \mathrm{~cm}^{-1}$ (2Dband), implying the existence of CNTs. ${ }^{50}$ In addition, the pure NiO spectrum shows two broad peaks at around $560 \mathrm{~cm}^{-1}$ and $1062 \mathrm{~cm}^{-1}$, which can be attributed to the first-(1P) and secondorder phonon (2P) scattering in NiO. ${ }^{51}$ All the characteristic peaks of CNTs and NiO can be observed from the CNT@NiO spectrum, confirming that the hybrids are composed of the CNTs and NiO nanosheets.

The more detailed morphology and structure of the CNTs, NiO and hierarchical CNT@NiO core-shell compounds were further analyzed using TEM and HRTEM. Fig. 3a shows that CNTs have a hollow tubular morphology feature and an outer diameter ranging between 20 and $60 \mathrm{~nm}$. As shown in Fig. 3b, the HRTEM image of the NiO nanosheets displays a distinct set of clear lattice fringes with interplanar spacings of around $0.21 \mathrm{~nm}$, corresponding to the (200) facet of the face-centered cubic phase of NiO. The selected-area electron diffraction (SAED) pattern (Fig. 3c) shows three intense rings that can be assigned to the (111), (200) and (220) crystal planes of NiO, in agreement with the XRD results. As can be clearly observed in Fig. 3d, the CNT@NiO hybrids exhibit a hierarchical core-shell structure and the surface of the CNTs is covered uniformly by a few layers of NiO shells. The high resolution TEM images (Fig. 3e and f) reveal that the NiO shells have an ultrathin structure, resulting in the full utilization of the active materials and ample exposed surfaces, which is advantageous for achieving excellent supercapacitive performance. In addition, the energy dispersive X-ray spectroscopy (EDS) spectrum (Fig. 3g) further demonstrates the elemental mapping results for the distribution of $\mathrm{C}, \mathrm{O}$ and Ni elements based on a single CNT@NiO core-shell nanosheet. As shown in Fig. 3h-j, the C, O and Ni elements exhibit a uniform and continuous distribution, corresponding to the hierarchical core-shell structure of the CNT@NiO composite. This reveals that the surfaces of the CNTs were successfully coated by $\mathrm{NiO}$ sheets, resulting in greatly shortened transport paths for both ions and electrons, which is beneficial for achieving a high rate capability. ${ }^{52}$

To test the electrochemical properties of the hierarchical CNT@NiO core-shell nanosheet electrodes, cyclic voltammetry (CV) and galvanostatic charge-discharge (GCD) techniques were applied, using a three-electrode configuration in $1 \mathrm{M} \mathrm{KOH}$ aqueous electrolyte. Fig. 4a compares the CV curves of the CNT, pure NiO, and CNT@NiO electrodes at a scan rate of $5 \mathrm{mV} \mathrm{s}^{-1}$ within a potential range of $0-0.6 \mathrm{~V}$. It can be clearly observed that the CV curves of the pure NiO and CNT@NiO electrodes consist of two strong redox peaks, indicating that the measured capacitance is mainly governed by a redox mechanism described as follows: ${ }^{53-56}$

$$
\mathrm{NiO}+\mathrm{OH}^{1-} \leftrightarrow \mathrm{NiOOH}+\mathrm{e}^{1-}
$$

which involves intercalation and deintercalation. ${ }^{33}$ Additionally, the CNT@NiO electrode exhibits a larger integral area than those of the individual CNT and NiO electrodes, according to the specific capacitance being proportional to the area of the CV curve, indicating a higher specific capacitance. Fig. 4b displays the CV curves of the CNT@NiO electrode at various scan rates ranging from 5 to $20 \mathrm{mV} \mathrm{s}^{-1}$. It can be observed that the shapes of these CV curves have no obvious distortion with increasing potential scan rate or even at ultrahigh scan rates, revealing an excellent rate capability and small equivalent series resistance. Galvanostatic charge-discharge (GCD) techniques were further employed to study electrochemical performance within the same potential window of $0-0.5 \mathrm{~V}$. Fig. 4c presents the contrasted GCD curves of the pure NiO and hierarchical CNT@NiO core-shell nanosheet electrodes at a current density of $1 \mathrm{~A} \mathrm{~g}^{-1}$, where the curves exhibit a triangular shape, indicating ideal capacitive behavior. Additionally, a longer discharge time is observed for the CNT@NiO electrode compared to the bare NiO electrode, implying that the specific capacitance has been greatly enhanced, and this result is in accordance with the CV curves. The GCD curves of the CNT@NiO electrode at different current densities are shown in Fig. 4d. The specific capacitance is calculated using the charge-discharge equation:

$$
C=I \times t /(m \times \Delta V),
$$

where $C$ is the specific capacitance, $I$ is the specific current, $t$ is the discharge time, $\Delta V$ is the potential window, and $m$ is the mass of active material. Fig. 4e shows the various specific current densities versus the specific capacitance. It can be seen that the specific capacitance decreases with increasing specific current. The CNT@NiO electrode exhibits an ultrahigh specific capacitance of $1000 \mathrm{~F} \mathrm{~g}^{-1}$ at $1 \mathrm{Ag}^{-1}$, which is higher than that of

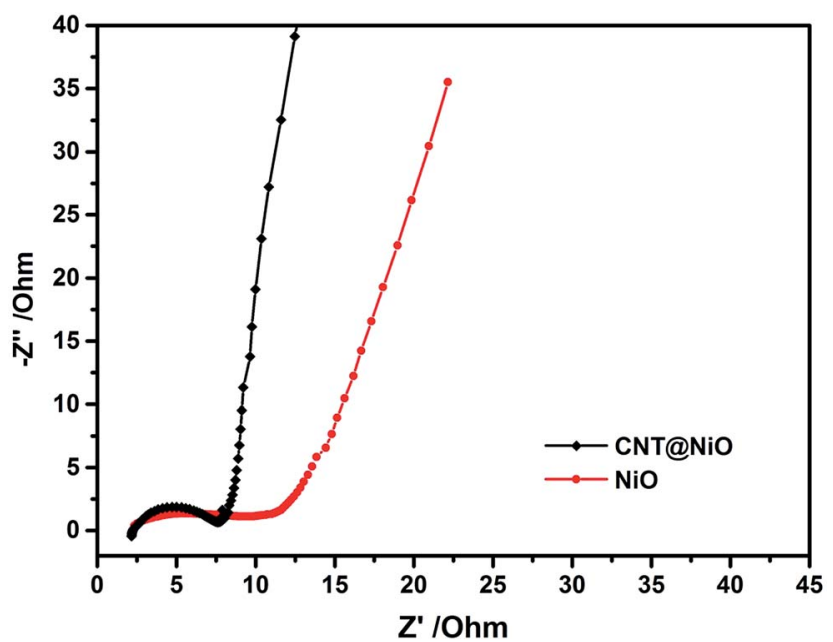

Fig. 5 Nyquist impedance plots of the $\mathrm{NiO}$ and $\mathrm{CNT} @ \mathrm{NiO}$ composites electrodes. 
pure $\mathrm{NiO}$ (298 $\mathrm{F} \mathrm{g}^{-1}$ at $1 \mathrm{~A} \mathrm{~g}^{-1}$ ). As a comparison, the specific capacitance of the CNT@NiO electrode retains a value of $600 \mathrm{~F}$ $\mathrm{g}^{-1}$ even at $20 \mathrm{~A} \mathrm{~g}^{-1}(55.56 \%$ capacitance retention), while the pure NiO electrode only retains a value of $76 \mathrm{~F} \mathrm{~g}^{-1}(26.7 \%$ capacitance retention) at the same current density, indicating that the CNT@NiO electrode shows a better rate capability. The

\section{(a) Purified graphene sheets}

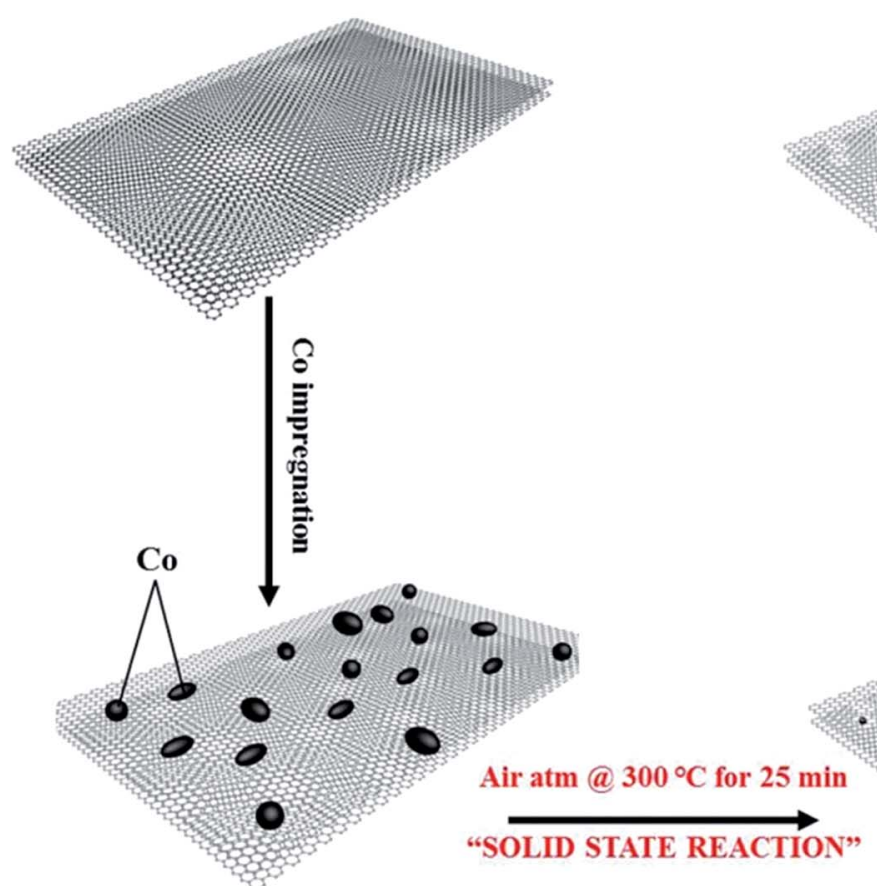

Co / Purified graphene sheets

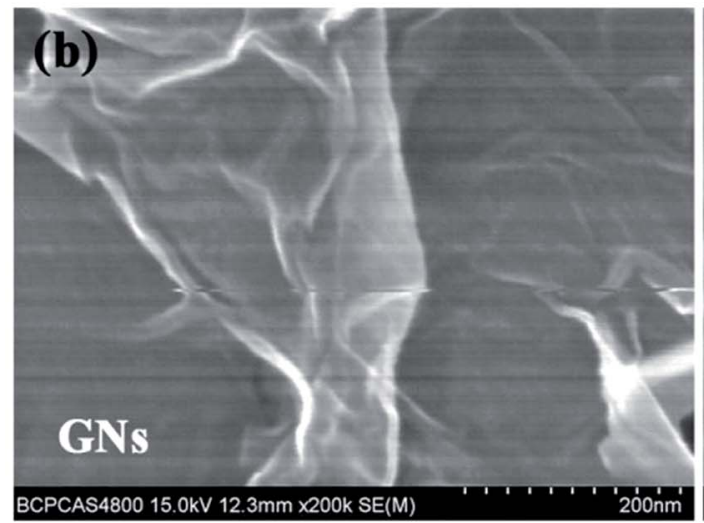

(d)

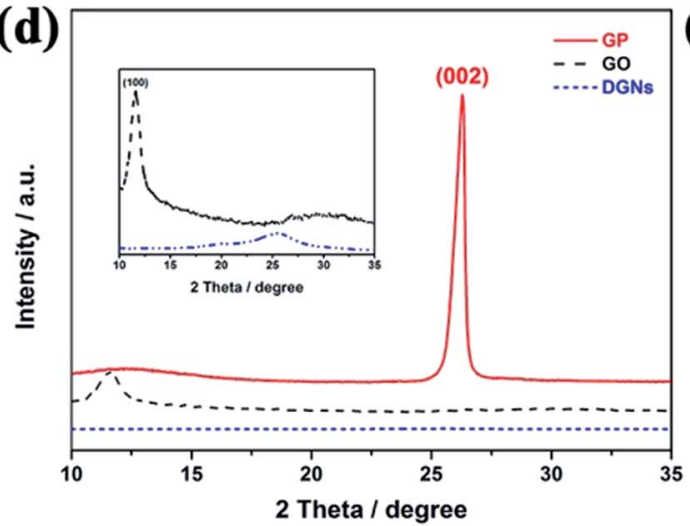

Defect-introduced graphene sheets

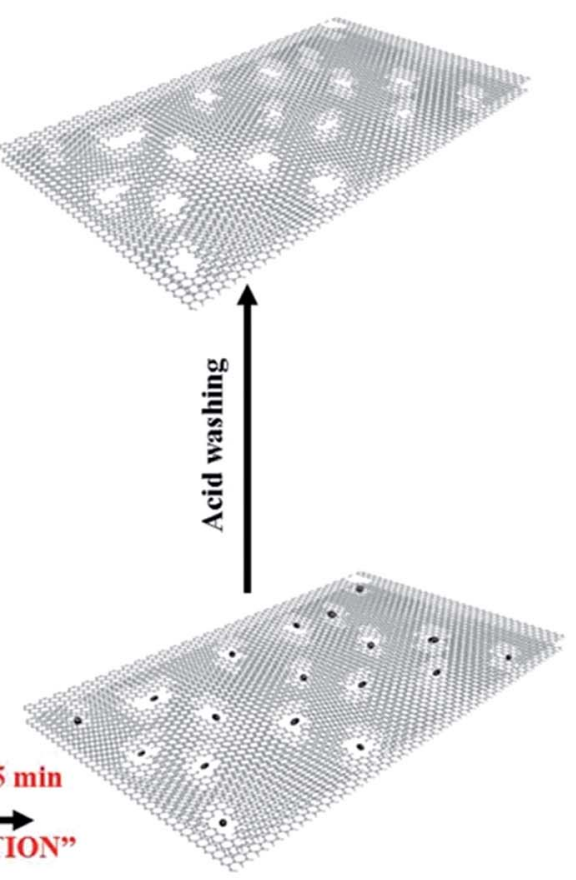

CoOx / Graphene sheets
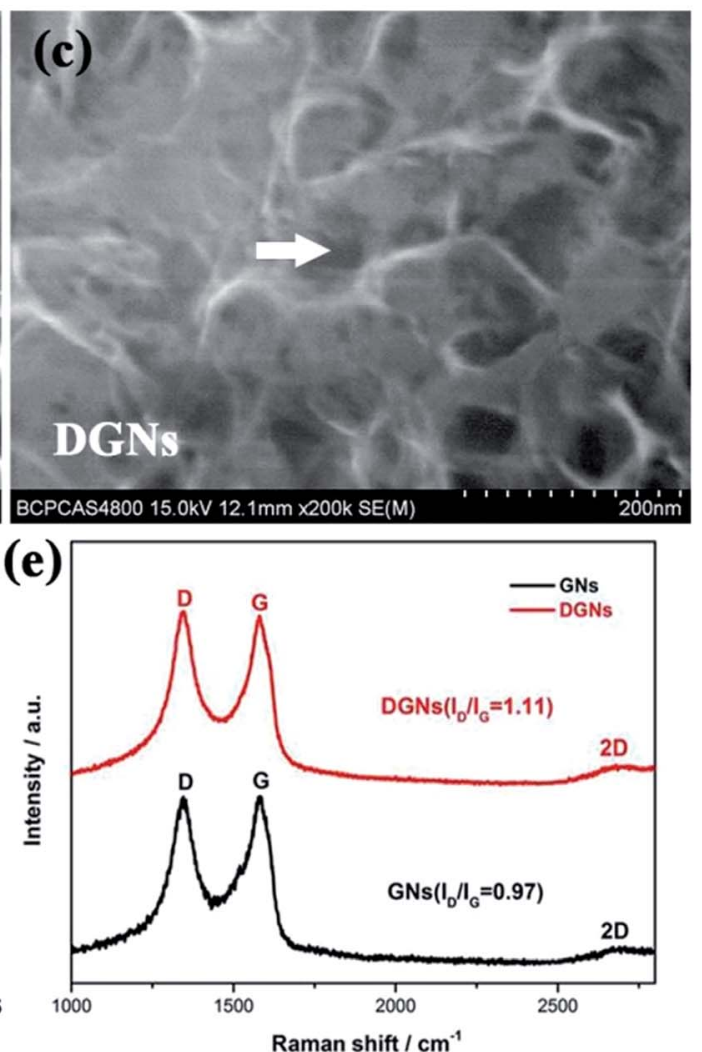

Fig. 6 (a) Schematic procedure for drilling DGNs. FESEM images of (b) GNs and (c) DGNs. (d) XRD patterns of GP, GO and DGNs and (e) Raman spectra of GNs and DGNs. 
remarkable capacitance characteristics of the CNT@NiO electrode may be due to the significant synergistic effect offered by the unique core-shell structure. Firstly, the CNTs in the hybrid offer both conductive channels and active interface centers, which would enable the good rate capability. Secondly, the ultrathin NiO sheets with large surface areas can effectively utilize the high pseudocapacitance and increase the chargetransport activity, thus favoring ionic movement during the discharging process. To evaluate the cycle reliability, the hierarchical CNT@NiO core-shell composite electrode and the pure $\mathrm{NiO}$ electrode were subjected to repeated charge-discharge cycles at the same constant current density of $10 \mathrm{~A} \mathrm{~g}^{-1}$ (Fig. 4f). It can be clearly observed that the specific capacitance of the CNT@NiO electrode slightly increases during the initial 650 cycles, which can be attributed to the activation process, resulting in the high utilization of the active material, and then the specific capacitance gradually decreases. ${ }^{57,58}$ After a continuous 3000 cycles, compared with the pure NiO electrode $(64.5 \%$ capacitance retained), $95.5 \%$ of the initial capacitance of the CNT@NiO electrode is retained. Thus the CNT@NiO electrode exhibits better cyclic stability. These results indicate that the hierarchical CNT@NiO core-shell hybrids hold great potential as a promising electrode material for high energy density ASC device applications.

To confirm that the electrical conductivity of $\mathrm{NiO}$ can be improved by incorporating the highly conductive frameworks of the CNTs, electrochemical impedance spectroscopy (EIS) experiments were carried out to analyze the NiO and CNT@NiO composite electrodes. Fig. 5 presents Nyquist plots, where the almost semicircular curve in the high frequency region is assigned to the interfacial charge-transfer resistance $\left(R_{\mathrm{ct}}\right)$, while the linear part in the low frequency region refers to the Warburg resistance $\left(W_{\mathrm{d}}\right)$, which represents the diffusion of ions in the electrolyte. ${ }^{59}$ It can be observed that the radius of the semicircle for the CNT@NiO composites is much smaller than that of NiO and the slope of the line for the CNT@NiO compounds is much greater than that of $\mathrm{NiO}$, revealing a lower diffusion resistance ( $R_{\mathrm{ct}}$ of NiO and CNT@NiO composite electrodes are $11.5 \Omega$ and 7 $\Omega$, respectively) and a better capacitor behavior. Due to the fact that the highly conductive CNTs provide adequate pathways for ions to reach the surface, thereby minimizing the ion diffusion length, the CNT@NiO composites display a better capacitive nature and more efficient charge transfer compared with NiO.

\subsection{Fabrication and characterization of negative electrode material}

Porous graphene has emerged as a promising material for applications in energy storage devices due to its excellent electrical conductivity, large surface area and appropriate pore structure. Herein, an electrode based on defect-introduced graphene sheets (DGNs) with hole structure was prepared using a facile and efficient cobalt catalyzed gasification strategy conducted at a relatively low temperature. ${ }^{48}$ Additionally, the prepared porous graphene exhibits ultrahigh specific capacity and good cyclic stability. Fig. 6a shows the fabrication process of the porous DGNs, which is schematically illustrated as a twostep process. The process involves the deposition of cobalt onto
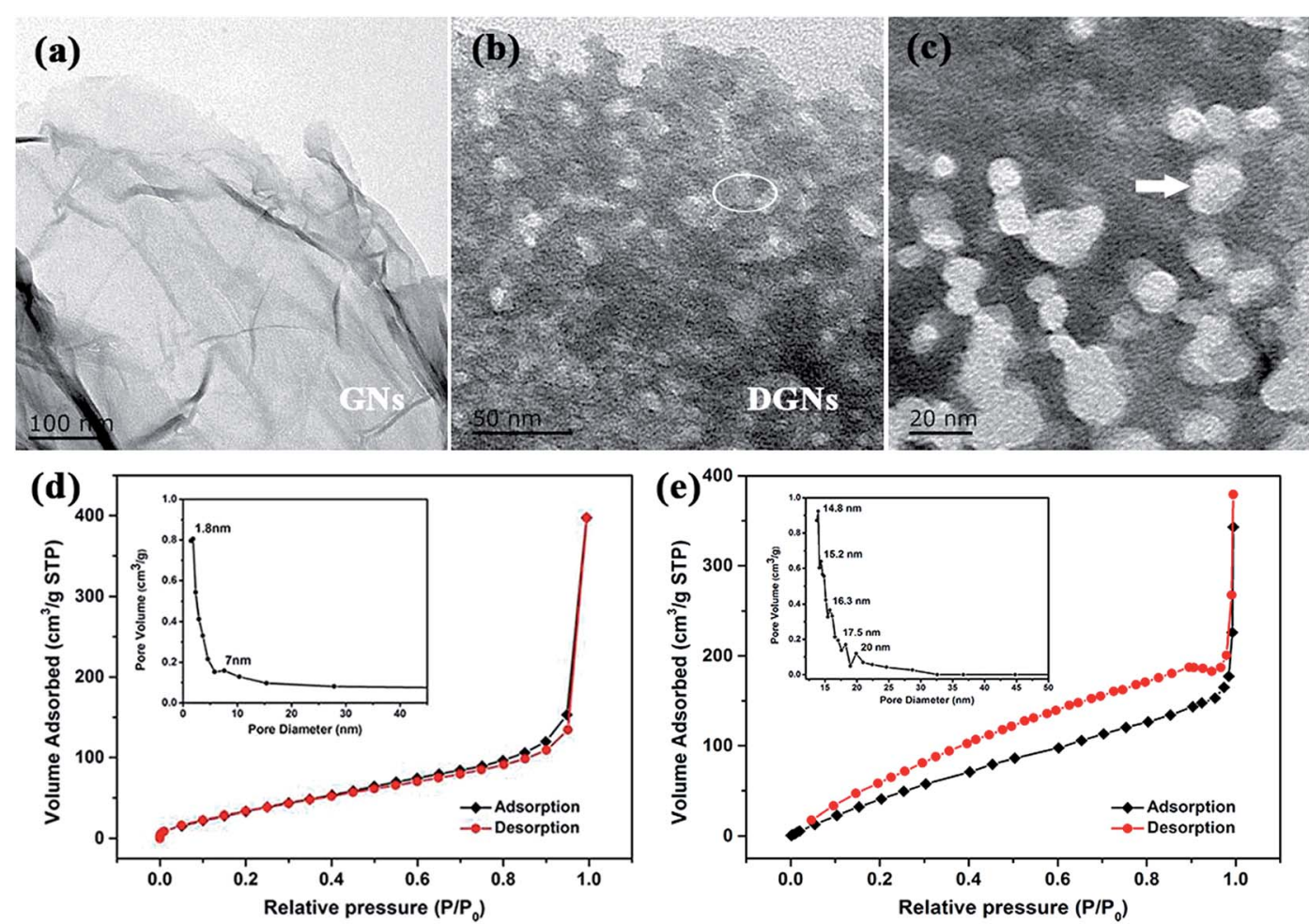

Fig. 7 TEM images of (a) GNs and (b and c) DGNs. (c) Magnified TEM image collected from the ringed region in (b). (d and e) $\mathrm{N}_{2}$ adsorptiondesorption isotherms of GNs and DGNs. 
the surfaces, and does not result in the occupation of the cavities in the graphene sheets. Cobalt then acts as the oxidation catalyst for the solid-state reaction and catalyzes the formation of hole structures on the exterior surfaces of the graphene sheets. The $\mathrm{CoO}_{x}$ nanoparticles are then removed by washing with acid, resulting in the DGNs. Fig. 6b exhibits a typical FESEM image of individual graphene sheets, showing almost transparent carbon nanosheets with a thin, wrinkled structure, which extend outwards with both sides exposed. The FESEM image in Fig. 6c shows that the DGNs have a well-defined porous structure, with an average pore size of about $15 \mathrm{~nm}$ and uniform pore size distribution. The phase information and structural changes that occur during the chemical processing in the transformation of original GNs to DGNs can be further characterized using XRD and Raman spectroscopy. As shown in the XRD patterns (Fig. 6d) of graphite powder (GP), graphite oxide (GO) and the DGNs, GP reveals a characteristic peak at $26.54^{\circ}$, which can be assigned to the (002) plane. Due to
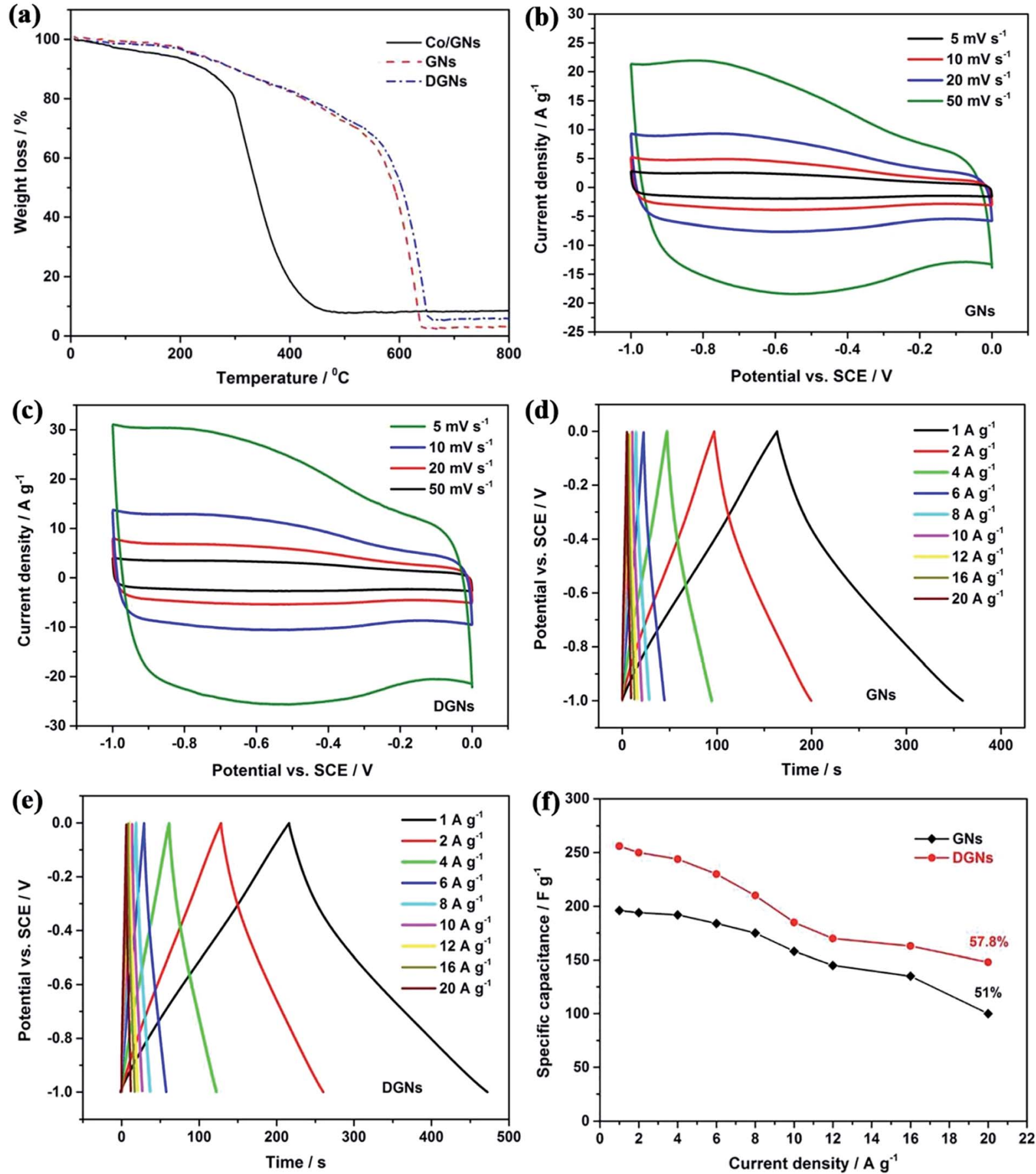

Fig. 8 TGA profiles (a) of DGNs, GNs and Co/GNs mixture. CV curves of (b) GNs and (c) DGNs at different scan rates. GCD curves of (d) GNs and (e) DGNs at various current densities. Specific capacitance of (f) GNs and DGNs at various different densities. 
oxidation, the (002) peak disappears and a new peak at $11.11^{\circ}$ identified as the (001) diffraction peak of GO appears. After the heat treatment under a nitrogen atmosphere, the diffraction peak of GO disappears, indicating that GO has been exfoliated to graphene. Meanwhile, the DGNs exhibit the same characteristic peak which all the carbon-based materials possess. Fig. 6e displays the Raman spectra of GNs and DGNs. In the Raman spectra, the peak for the G-band at about $1587 \mathrm{~cm}^{-1}$ due to the doubly degenerated zone center $\mathrm{E}_{2 \mathrm{~g}}$ mode, and the peak for the D-band at about $1352 \mathrm{~cm}^{-1}$ characterizing the disordered $\mathrm{sp}^{2}$ graphene are present. ${ }^{50,51}$ The peak intensity ratio between the two bands $\left(I_{\mathrm{D}} / I_{\mathrm{G}}\right)$ is often considered a measurement of the graphene quality. Obviously, the $\mathrm{D} / \mathrm{G}$ intensity ratio for the DGNs $\left(I_{\mathrm{D}} / I_{\mathrm{G}}=1.11\right)$ is stronger than that for the GNs $\left(I_{\mathrm{D}} /\right.$ $\left.I_{\mathrm{G}}=0.97\right)$, indicating the existence of defect structures in the DGNs which come from the holes on the sidewalls due to the Co catalyzed defect introducing process. ${ }^{57}$

The crystal structure was further determined using TEM. As shown in Fig. 7a, the GNs show a thin wrinkled paper-like curled morphology and the exterior walls of the GNs display hole-free and well-ordered graphitic crystallites. On the contrary, Fig. $7 \mathrm{~b}$ and $\mathrm{c}$ show that hole structures have been introduced on the surfaces of the GNs after the oxidation of the $\mathrm{Co} / \mathrm{GNs}$ and acid digestion process. The Co/GNs mixture undergo heating in air atmosphere at $300{ }^{\circ} \mathrm{C}$ for $25 \mathrm{~min}$ to trigger cobalt catalyzed gasification strategy. The magnified TEM image of the DGNs in Fig. 7c clearly displays abundant micro-/mesopores and there are two pivotal factors that affect the defect structure, which control the size and prevent the agglomeration of $\mathrm{CoO}_{x}$ particles. The pore structure of the prepared DGNs was analyzed using $\mathrm{N}_{2}$ adsorption-desorption isotherms. As shown in Fig. 7d and e, the isotherm of the GNs (Fig. 7d) is of type II in IUPAC level and has a rapid agglomeration step as the relative pressure approaches unity. ${ }^{60}$ Additionally, the isotherm of the GNs displays the largest saturated adsorption amount at $P / P_{0}=1$, corresponding to the multilayer filling of large pores, which is responsible for adsorption of a large quantity of $\mathrm{N}_{2}$ at high pressure. In contrast, the isotherm of the prepared DGNs is close to type IV, possessing an obvious hysteresis loop, which indicates the obviously mesoporous character of the sample. The Barrett-Joyner-Halenda (BJH) method was performed to further analyze the porosity and pore size distribution. The inset of Fig. 7d reveals that the GNs have a wide distribution of pore sizes, with average pore sizes of 1.8 , 5.3 and $7 \mathrm{~nm}$, revealing a main distribution of micropores. Unlike in the GNs, mesopores with maximum sizes of 14.8, 17.5 and $20 \mathrm{~nm}$ are abundantly concentrated in the DGNs, implying that the pore sizes distribution extends to the mesopore-range (inset of Fig. 7e). In view of the above analysis, the BET surface areas of the GNs and DGNs are up to 195 and $456 \mathrm{~m}^{2}$ $\mathrm{g}^{-1}$, and the total pore volume of the GNs and DGNs are up to 0.21 and $0.47 \mathrm{~cm}^{3} \mathrm{~g}^{-1}$, respectively.

Thermogravimetric analysis (TGA) was employed to determine the oxidation profile of the cobalt-assisted carbon gasification used for defect formation. Fig. 8a shows that each curve has an initial mass loss at a relatively low temperature $\left(<100{ }^{\circ} \mathrm{C}\right)$, owing to the evaporation of physisorbed water. In contrast, the
Co/GN mixture exhibits a faster weight loss and is oxidized completely at a lower oxidation temperature than that of the GNs and DGNs. In particular, cobalt oxide catalyzes the oxidation of carbon by offering lattice oxygen and promoting $\mathrm{C}-\mathrm{O}$ formation to release CO gas. ${ }^{61}$ The process is as follows:

$$
6 \mathrm{CoO}+\mathrm{O}_{2} \rightarrow 2 \mathrm{Co}_{3} \mathrm{O}_{4}
$$

and

$$
\mathrm{Co}_{3} \mathrm{O}_{4}+\mathrm{C} \rightarrow 3 \mathrm{CoO}+\mathrm{CO}
$$

which results in the formation of hole structures on the graphene sheet surfaces with some $\mathrm{CoO}_{x}$ nanoparticles still residing in the holes, followed by Co removal through acid digestion. The open hole structures on the surfaces of graphene sheets remain. Additionally, the formation of hole structures demonstrates that only carbon in contact with $\mathrm{CoO}_{x}$ can be oxidized during the solid-state process, leaving the other parts unaffected, resulting in the retention of the planar $\mathrm{sp}^{2}$ carbon sheets and high electrical conductivity.

Fig. $8 \mathrm{~b}$ and $\mathrm{c}$ show the comparison of the CV curves of the GN and DGN electrodes at different scan rates ranging from 5 to $50 \mathrm{mV} \mathrm{s}^{-1}$ using $1 \mathrm{M} \mathrm{KOH}$ as the electrolyte. It can be clearly observed that the CV curves of the DGN electrode show a larger integral area with an almost rectangular shape, compared with those of the GN electrode at the same scan rate, suggesting that the DGNs possess ideal capacitance and superior charge transport. Typical GCD curves were obtained for the GN and DGN electrodes with a potential range between -1 to $0 \mathrm{~V}$ (Fig. 8d and e), in which all the GCD curves are nearly triangular in shape even at an ultrahigh current density of $20 \mathrm{~A} \mathrm{~g}^{-1}$, indicating good reversibility. In addition, there is no obvious $i R$ drop in any of the curves, revealing a low internal resistance. Fig. 8f displays the specific capacitance of the GN and DGN electrodes at various current densities. Compared with the GN electrode (196 $\mathrm{F} \mathrm{g}^{-1}$ at $1 \mathrm{~A} \mathrm{~g}^{-1}, 100 \mathrm{~F} \mathrm{~g}^{-1}$ at $1 \mathrm{~A} \mathrm{~g}^{-1}$ ), the DGN electrode shows a higher specific capacitance of $256 \mathrm{~F} \mathrm{~g}^{-1}$ at $1 \mathrm{~A}$ $\mathrm{g}^{-1}$. Even at $20 \mathrm{~A} \mathrm{~g}^{-1}$, a specific capacitance of $148 \mathrm{~F} \mathrm{~g}^{-1}$ is still achieved, indicating a more excellent capacitance stability

Table 1 Comparison of specific capacitance for various carbon materials in aqueous electrolytes reported in the literature

\begin{tabular}{lllll}
\hline Material & Electrolyte & $\begin{array}{l}\text { Specific } \\
\text { capacitance } \\
\left(\mathrm{F} \mathrm{g}^{-1}\right)\end{array}$ & $\begin{array}{l}\text { Current } \\
\text { density } \\
\left(\mathrm{A} \mathrm{g}^{-1}\right)\end{array}$ & Ref. \\
\hline NG-900 & $6 \mathrm{M} \mathrm{KOH}$ & 130 & 0.5 & 62 \\
aNG & $1 \mathrm{M} \mathrm{KOH}$ & 132.4 & 0.1 & 63 \\
TsG & $6 \mathrm{M} \mathrm{KOH}$ & 180 & 0.5 & 64 \\
AC800NH & $6 \mathrm{M} \mathrm{KOH}$ & 196 & 1 & 65 \\
HPCFs & $6 \mathrm{M} \mathrm{KOH}$ & 206 & 1 & 66 \\
C-900 & $6 \mathrm{M} \mathrm{KOH}$ & 210 & 1 & 67 \\
PANecoal- & $6 \mathrm{M} \mathrm{KOH}$ & 230 & 1 & 68 \\
AC5 & & & & \\
AC800 & $7 \mathrm{M} \mathrm{KOH}$ & 232 & 0.05 & 69 \\
PCPs & $1 \mathrm{M} \mathrm{KOH}$ & 245 & 1 & 70 \\
DGNs & $1 \mathrm{M} \mathrm{KOH}$ & 256 & 1 & This \\
& & & & work
\end{tabular}


(57.8\% retention relative to $\left.1 \mathrm{~A} \mathrm{~g}^{-1}\right)$. Table 1 summarizes the specific capacitance of previously reported carbon-based material supercapacitor electrodes. ${ }^{62-70}$ Obviously, the electrochemical performance of our DGN electrode is higher than those of these reported electrodes. Such excellent electrochemical performance can be attributed to the appropriate pore structure, large specific surface area and good electrical conductivity. Through this strategy, the porous structure has shown many merits. Firstly, such a porous structure greatly increases the electrode/electrolyte contact area, resulting in the further enhancement of the capacitive performance. Secondly, the porous structure offers a high packing density, which magnifies the volume energy density of the electrode material. Thirdly, the porous structure provides vast pathways for the electron collection/transport between the overlapping DGN, which is important for the enhancement of the high-rate (a)

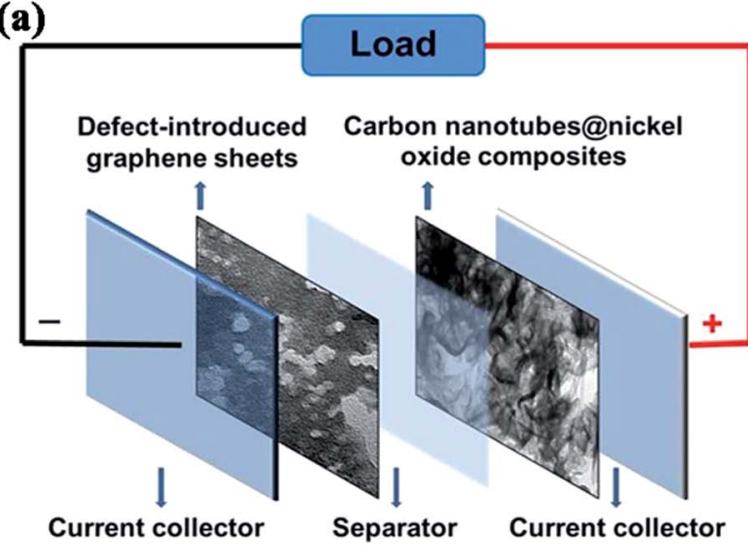

Electrolyte: $1 \mathrm{M} \mathrm{KOH}$

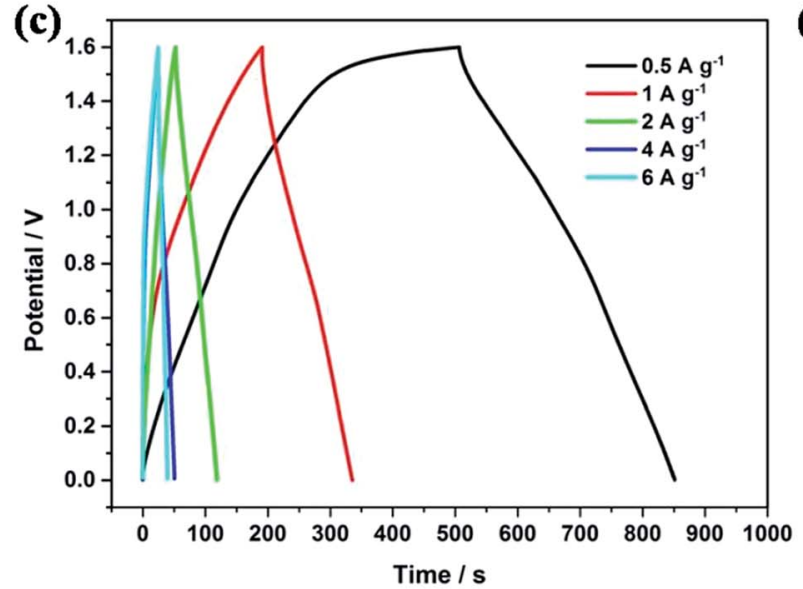

(e)

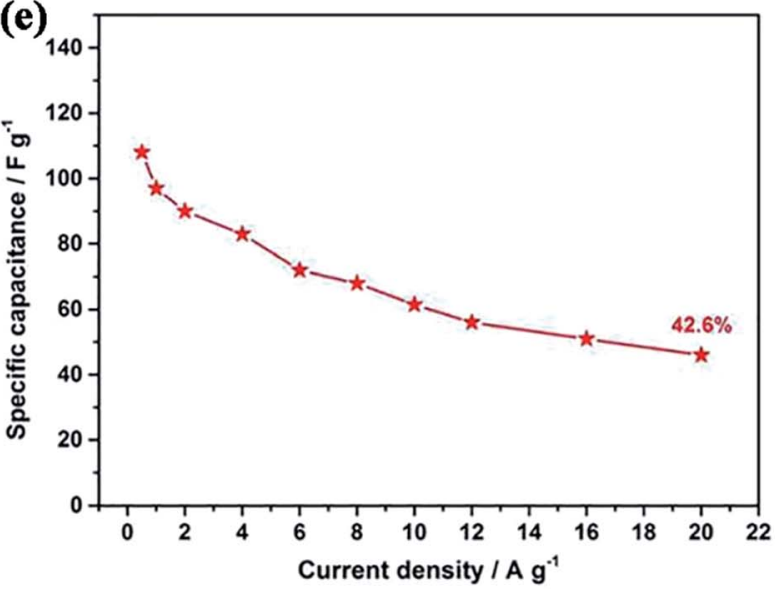

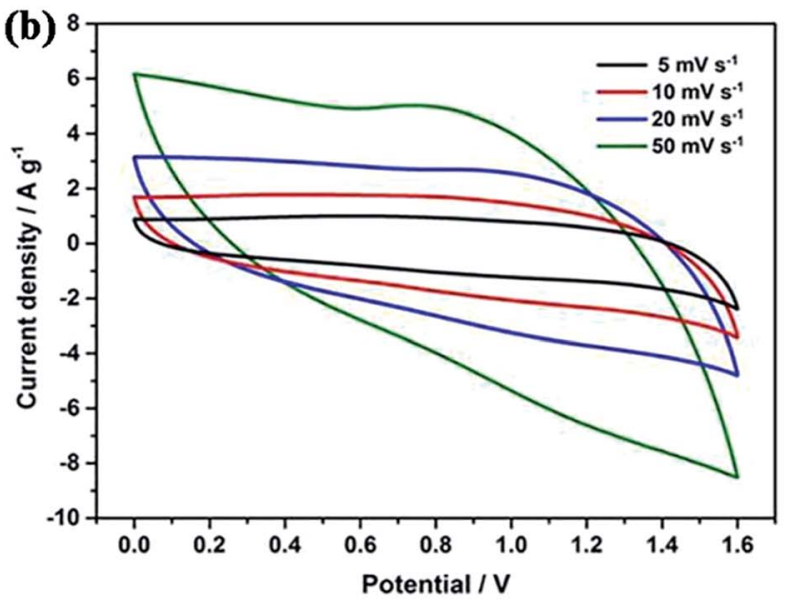
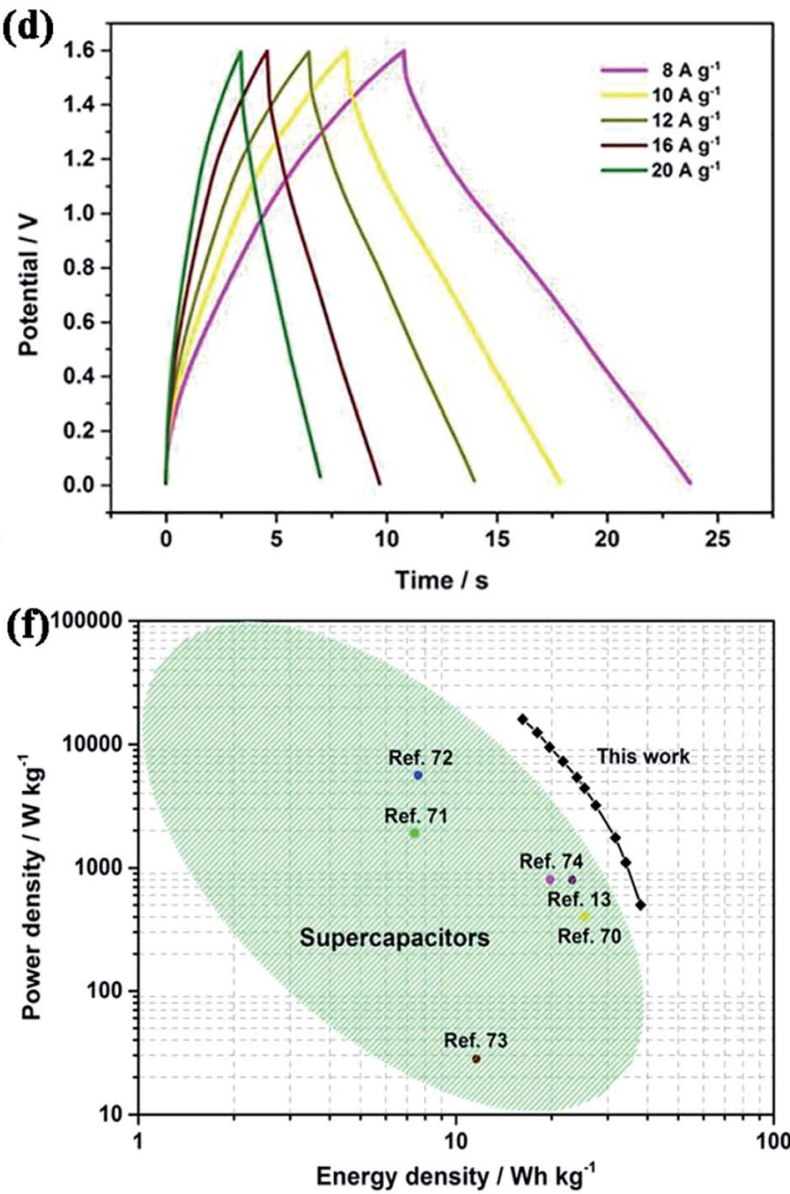

Fig. 9 (a) Schematic illustration of the assembled structure of the CNTaNiO//DGNs ASC device. (b) CV curves of CNTaNiO//DGNs ASC at different scan rates. (c and d) GCD curves of CNT@NiO//DGNs ASC at various current densities. (e) Specific capacitance of CNTaNiO//DGNs ASC at different densities. (f) Ragone plot of the CNT@NiO//DGNs ASC, compared with many previously reported asymmetric supercapacitors. 
performance. Thanks to the unique structural features, such as ample mesopore distribution and open flat layers with ultrahigh surface area, the DGN electrode displays excellent kinetic electrochemical capability as well as stable profiles and good reversibility in energy storage cell applications.

\subsection{Electrochemical performance of the CNT@NiO//DGNs ASC device}

To increase the operating voltage and evaluate the electrochemical properties of the prepared samples, an ASC device was constructed using a negative DGN electrode and a positive hierarchical CNT@NiO core-shell nanosheet electrode with one piece of cellulose paper as the separator in $1 \mathrm{M} \mathrm{KOH}$ electrolyte. The device is denoted as the CNT@NiO//DGNs ASC device (Fig. 9a). For an ASC, the charge $(Q)$ stored by two electrodes should follow the two equations of

$$
Q_{+}=Q_{-}
$$

and

$$
Q=C \times \Delta V \times m
$$

to obtain the maximum performance. Where $C$ represents the specific capacitance, $\Delta V$ is the potential range of the chargedischarge process and $m$ is the mass of activated material. In our work, the value of $C_{-} / C_{+}$was found to be 0.25 at $1 \mathrm{~A} \mathrm{~g}^{-1}$ and the value of $\Delta V_{-} / \Delta V_{+}$was found to be 2 . Based on these data, the optimal mass ratio of the two electrodes should be $m_{\text {(CNT@NiO) }} /$ $m_{\text {(DGNs) }}=0.5$. Taking advantage of the wide potential range of the two electrodes, it was expected that the operating potential of our CNT@NiO//DGNs ASC device can be extended to $1.6 \mathrm{~V}$. Fig. 9b displays a typical CV experiment of the ASC at different scan rates from 5 to $50 \mathrm{mV}^{-1}$. All the curves exhibit a nearly rectangular shape with well-defined redox peaks, illustrating the remarkable pseudocapacitive nature and good rate stability of our device. As shown in Fig. 9c and d, galvanostatic chargedischarge curves of the ASC at a series of current densities from 0.5 to $20 \mathrm{~A} \mathrm{~g}^{-1}$ were obtained. There is no clear $i R$ drop observed in any of the discharge curves, indicating the fast transfer of electrons and transport of electrolyte ions in the ASC. Additionally, all the charge-discharge curves are almost symmetrical, revealing good electrochemical reversibility and good charge-discharge stability. Fig. 9e summarizes the specific capacitance of our ASC device obtained from its chargedischarge curves (Fig. 9d) and the specific capacitance decreases gradually with increasing current density. The ASC device yields a maximum specific capacitance of $108 \mathrm{~F} \mathrm{~g}^{-1}$ at 0.5 $\mathrm{A} \mathrm{g}^{-1}$, and even at a specific current of $20 \mathrm{~A} \mathrm{~g}^{-1}$, the specific capacitance still retains a value of $46 \mathrm{~F} \mathrm{~g}^{-1}$. Fig. 9f shows a Ragone plot depicting the relationship between the power density and energy density for the CNT@NiO//DGNs ASC device, displaying a maximum energy density of $38.1 \mathrm{~W} \mathrm{~h} \mathrm{~kg}^{-1}$ at a power density of $500 \mathrm{~W} \mathrm{~kg}^{-1}$. Even at an ultrahigh power density of $16 \mathrm{~kW} \mathrm{~kg}^{-1}$, the energy density can still retain a value of $16.2 \mathrm{~W} \mathrm{~h} \mathrm{~kg}^{-1}$. This excellent electrochemical performance exhibited by our ASC device exceeds the performance of most previously reported Ni-based ASCs, such as Ni-Co oxide//
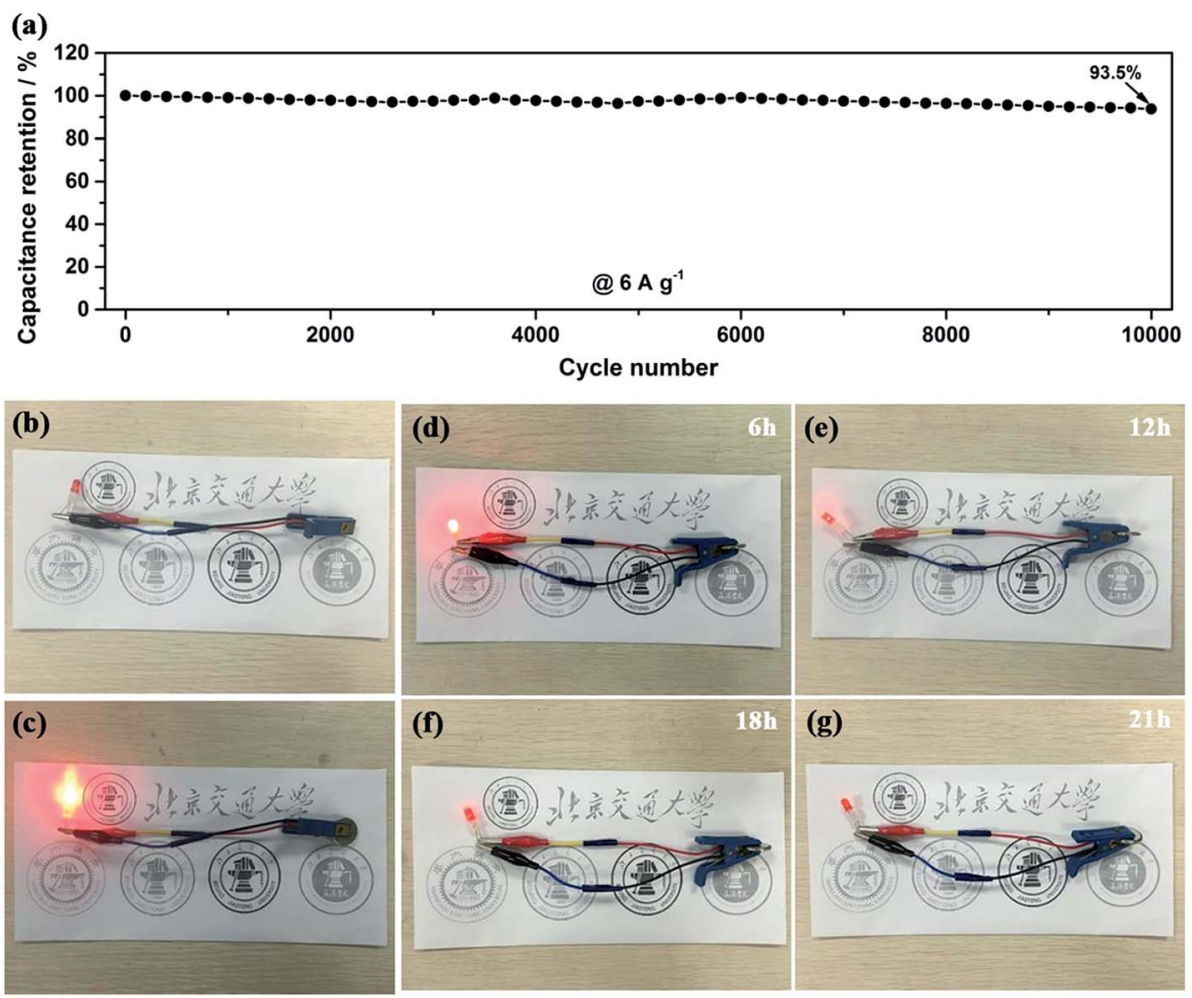

Fig. 10 Cycling performance (a) of the CNT@NiO//DGNs ASC device at a current density of $6 \mathrm{Ag}^{-1}$. (b-g) Photographs of the CNT aNiO//DGNs ASC device powering a red LED indicator and the red LED at different stages. 
activated carbon $\left(7.4 \mathrm{~W} \mathrm{~h} \mathrm{~kg}{ }^{-1}\right.$ at $\left.1903 \mathrm{~W} \mathrm{~kg}^{-1}\right){ }^{71}$ graphene$\mathrm{NiCo}_{2} \mathrm{O}_{4} / /$ activated carbon $\left(7.6 \mathrm{~W} \mathrm{~h} \mathrm{~kg}^{-1}\right.$ at $\left.5600 \mathrm{~W} \mathrm{~kg}^{-1}\right){ }^{72}$ $\mathrm{NiO} / /$ carbon (11.6 $\mathrm{W} \mathrm{h} \mathrm{kg}{ }^{-1}$ at $28 \mathrm{~W} \mathrm{~kg}^{-1}$ ), ${ }^{73} \mathrm{Ni}_{3} \mathrm{~S}_{2} /$ carbon//AC $\left(19.8 \mathrm{~W} \mathrm{~h} \mathrm{~kg}{ }^{-1}\right.$ at $\left.798 \mathrm{~W} \mathrm{~kg}^{-1}\right),{ }^{74}$ graphene hydrogel $/ / \mathrm{MnO}_{2}-$ nickel foam (23.2 $\mathrm{W} \mathrm{h} \mathrm{kg}^{-1}$ at $\left.800 \mathrm{~W} \mathrm{~kg}^{-1}\right){ }^{13}$ and CNT@NiO// porous carbon polyhedrons $\left(25.4 \mathrm{~W} \mathrm{~h} \mathrm{~kg}^{-1}\right.$ at $\left.400 \mathrm{~W} \mathrm{~kg}^{-1}\right) .^{70}$ The outstanding electrochemical performance of our ASC device, due to the wide voltage window and relatively small leakage current, stems from the strong synergistic effect between the two electrodes.

Another important key factor of the ASC device is its cycling performance, as shown in Fig. 10a. Consecutive galvanostatic charge-discharge cycling at a current density of $6 \mathrm{~A} \mathrm{~g}^{-1}$ for 10000 cycles was performed to evaluate the cycling stability. After 10000 cycles, 94\% of the initial capacitance is still retained and the shape of the GCD curves are not obviously changed, demonstrating a relatively small leakage current and the low self-discharge characteristics of our ASC device. To investigate the practical application of our ASC cell as an efficient power supply, it was applied to light a red-light LED indicator. As shown in Fig. 10b-g, the red LED remains continuously bright for $21 \mathrm{~h}$. All the outstanding electrochemical properties of the device suggest that our electrode materials are suitable for potential applications in highperformance energy storage devices.

\section{Conclusions}

In this work, hierarchical CNT@NiO core-shell nanosheets were developed via a facile chemical bath deposition method, combined with a thermal annealing approach, and the DGNs with hole structure were synthesized utilizing an efficient cobalt catalyzed gasification process. By taking advantage of the unique nanostructure, the CNT@NiO compound electrode demonstrates ultrahigh specific capacitance $\left(1000 \mathrm{~F} \mathrm{~g}^{-1}\right.$ at $1 \mathrm{~A}$ $\mathrm{g}^{-1}$ ), superior to the pure NiO electrode, and the DGN electrode exhibits a specific capacitance of up to $256 \mathrm{~F} \mathrm{~g}^{-1}$ at $1 \mathrm{~A} \mathrm{~g}^{-1}$. Thanks to the wide voltage window and the high synergistic effects between the two electrodes, an advanced asymmetric supercapacitor based on hierarchical CNT@NiO core-shell nanosheets as the positive electrode and DGNs as the negative electrode was fabricated and tested in a $1.6 \mathrm{~V}$ potential window. The CNT@NiO//DGNs ASC device delivers a maximum energy density of $38.1 \mathrm{~W} \mathrm{~h} \mathrm{~kg}^{-1}$ and power density of $16000 \mathrm{~W} \mathrm{~kg}^{-1}$, as well as an excellent cycling stability ( $94 \%$ capacitance retention after 10000 cycles). Herein, our work provides a novel strategy to construct porous graphene and proves that the CNT@NiO// DGNs ASC device is a promising candidate for energy storage equipment applications. This work should propel the research progress of graphene-based materials as ideal electrode materials for supercapacitors in practical use.

\section{Acknowledgements}

We are grateful for the financial support of the National Basic Research Program (2016YFA0202300, 2016YFA0202302), the National Natural Science Fund Project (under Contract no.
61527817, 61335006, 61378073) and the Beijing Science and Technology Committee (Z151100003315006).

\section{References}

1 A. Balducci, R. Dugas, P. L. Taberna, P. Simon, D. Plée, M. Mastragostino and S. Passerini, J. Power Sources, 2007, 165, 922-927.

2 D. A. C. Brownson, D. K. Kampouris and C. E. Banks, J. Power Sources, 2011, 196, 4873-4885.

3 C. Du and N. Pan, Nanotechnology, 2006, 17, 5314-5318.

4 Z. Hu, X. Xiao, C. Chen, T. Li, L. Huang, C. Zhang, J. Su, L. Miao, J. Jiang, Y. Zhang and J. Zhou, Nano Energy, 2015, 11, 226-234.

5 R. Bendi, V. Kumar, V. Bhavanasi, K. Parida and P. S. Lee, Adv. Energy Mater., 2016, 6, 1501833.

6 B. E. Conway and W. G. Pell, J. Solid State Electrochem., 2003, 7, 637-644.

7 J. Yan, Z. Fan, W. Sun, G. Ning, T. Wei, Q. Zhang, R. Zhang, L. Zhi and F. Wei, Adv. Funct. Mater., 2012, 22, 2632-2641.

8 C. Liu, F. Li, L. P. Ma and H. M. Cheng, Adv. Mater., 2010, 22, E28-E62.

9 M. Acerce, D. Voiry and M. Chhowalla, Nat. Nanotechnol., 2015, 10, 313-318.

10 J. J. Yoo, K. Balakrishnan, J. Huang, V. Meunier, B. G. Sumpter, A. Srivastava, M. Conway, A. L. Reddy, J. Yu, R. Vajtai and P. M. Ajayan, Nano Lett., 2011, 11, 1423-1427.

11 S. T. Senthilkumar, Y. Wang and H. Huang, J. Mater. Chem. A, 2015, 3, 20863-20879.

12 Y. Huang, H. Hu, Y. Huang, M. Zhu, W. Meng, C. Liu, Z. Pei, C. Hao, Z. Wang and C. Zhi, ACS Nano, 2015, 9, 4766-4775.

13 H. Gao, F. Xiao, C. B. Ching and H. Duan, ACS Appl. Mater. Interfaces, 2012, 4, 2801-2810.

14 A. Izadi-Najafabadi, S. Yasuda, K. Kobashi, T. Yamada, D. N. Futaba, H. Hatori, M. Yumura, S. Iijima and K. Hata, Adv. Mater., 2010, 22, E235-E241.

15 W. G. Pell and B. E. Conway, J. Power Sources, 2004, 136, 334-345. 16 L. Demarconnay, E. Raymundo-Piñero and F. Béguin, J. Power Sources, 2011, 196, 580-586.

17 J. Liu, J. Jiang, C. Cheng, H. Li, J. Zhang, H. Gong and H. J. Fan, Adv. Mater., 2011, 23, 2076-2081.

18 M. Yu, Z. Wang, Y. Han, Y. Tong, X. Lu and S. Yang, J. Mater. Chem. A, 2016, 4, 4634-4658.

19 L. Shen, L. Yu, H. B. Wu, X. Y. Yu, X. Zhang and X. W. Lou, Nat. Commun., 2015, 6, 6694.

20 B. Wang, T. Zhu, H. B. Wu, R. Xu, J. S. Chen and X. W. Lou, Nanoscale, 2012, 4, 2145-2149.

21 X. Yu, B. Lu and Z. Xu, Adv. Mater., 2014, 26, 1044-1051.

22 X. H. Xia, J. P. Tu, Y. Q. Zhang, Y. J. Mai, X. L. Wang, C. D. Gu and X. B. Zhao, J. Phys. Chem. C, 2011, 115, 22662-22668.

23 F. Luan, G. Wang, Y. Ling, X. Lu, H. Wang, Y. Tong, X. X. Liu and Y. Li, Nanoscale, 2013, 5, 7984-7990.

24 Z. Yang, F. Xu, W. Zhang, Z. Mei, B. Pei and X. Zhu, J. Power Sources, 2014, 246, 24-31.

25 S. I. Kim, J. S. Lee, H. J. Ahn, H. K. Song and J. H. Jang, ACS Appl. Mater. Interfaces, 2013, 5, 1596-1603.

26 Y. Zou and Y. Wang, Nanoscale, 2011, 3, 2615-2620. 
27 W. Wen, J.-M. Wu and M.-H. Cao, J. Mater. Chem. A, 2013, 1, 3881.

28 X. Xu, H. Tan, K. Xi, S. Ding, D. Yu, S. Cheng, G. Yang, X. Peng, A. Fakeeh and R. V. Kumar, Carbon, 2015, 84, 491-499.

29 X. Xu, J. Liang, H. Zhou, S. Ding and D. Yu, RSC Adv., 2014, 4, 3181-3187.

30 K.-W. Nam, K.-H. Kim, E.-S. Lee, W.-S. Yoon, X.-Q. Yang and K.-B. Kim, J. Power Sources, 2008, 182, 642-652.

31 Y. Qian, R. Liu, Q. Wang, J. Xu, D. Chen and G. Shen, J. Mater. Chem. A, 2014, 2, 10917.

32 B. Zhao, J. Song, P. Liu, W. Xu, T. Fang, Z. Jiao, H. Zhang and Y. Jiang, J. Mater. Chem., 2011, 21, 18792.

33 H. Wang, H. Yi, X. Chen and X. Wang, Electrochim. Acta, 2013, 105, 353-361.

34 M. F. De Volder, S. H. Tawfick, R. H. Baughman and A. J. Hart, Science, 2013, 339, 535-539.

35 C. de las Casas and W. Li, J. Power Sources, 2012, 208, 74-85. 36 J. M. Schnorr and T. M. Swager, Chem. Mater., 2011, 23, 646-657.

37 M. D. Stoller and R. S. Ruoff, Energy Environ. Sci., 2010, 3, 1294.

38 C. N. Rao, A. K. Sood, K. S. Subrahmanyam and A. Govindaraj, Angew. Chem., 2009, 48, 7752-7777.

39 Y. Huang, J. Liang and Y. Chen, Small, 2012, 8, 1805-1834.

40 Y. Shao, J. Wang, H. Wu, J. Liu, I. A. Aksay and Y. Lin, Electroanalysis, 2010, 22, 1027-1036.

41 X. Ji, K. T. Lee and L. F. Nazar, Nat. Mater., 2009, 8, 500-506.

42 D. S. Jung, T. H. Hwang, J. H. Lee, H. Y. Koo, R. A. Shakoor, R. Kahraman, Y. N. Jo, M. S. Park and J. W. Choi, Nano Lett., 2014, 14, 4418-4425.

43 M. Q. Zhao, Q. Zhang, J. Q. Huang, G. L. Tian, J. Q. Nie, H. J. Peng and F. Wei, Nat. Commun., 2014, 5, 3410.

44 G. Wang, X. Shen, J. Yao and J. Park, Carbon, 2009, 47, 20492053.

45 S. Pang, H. N. Tsao, X. Feng and K. Müllen, Adv. Mater., 2009, 21, 3488-3491.

46 S. C. Eagle and G. K. Fedder, Appl. Phys. Lett., 1999, 74, 39023903.

47 Z. Chen, Y.-M. Lin, M. J. Rooks and P. Avouris, Phys. E, 2007, 40, 228-232.

48 H. S. Oktaviano, K. Yamada and K. Waki, J. Mater. Chem., 2012, 22, 25167.

49 B. Gao, C.-z. Yuan, L.-h. Su, L. Chen and X.-g. Zhang, J. Solid State Electrochem., 2008, 13, 1251-1257.

50 A. C. Ferrari, J. C. Meyer, V. Scardaci, C. Casiraghi, M. Lazzeri, F. Mauri, S. Piscanec, D. Jiang, K. S. Novoselov, S. Roth and A. K. Geim, Phys. Rev. Lett., 2006, 97, 187401.

51 H. Wang, H. Yi, X. Chen and X. Wang, J. Mater. Chem. A, 2014, 2, 3223.

52 D. Sun, Q. Zou, Y. Wang, Y. Wang, W. Jiang and F. Li, Nanoscale, 2014, 6, 6557-6562.
53 B. Wang, J. S. Chen, Z. Wang, S. Madhavi and X. W. D. Lou, Adv. Energy Mater., 2012, 2, 1188-1192.

54 X. Cao, Y. Shi, W. Shi, G. Lu, X. Huang, Q. Yan, Q. Zhang and H. Zhang, Small, 2011, 7, 3163-3168.

55 K. S. Novoselov, A. K. Geim, S. V. Morozov, D. Jiang, Y. Zhang, S. V. Dubonos, I. V. Grigorieva and A. A. Firsov, Science, 2004, 306, 666-669.

56 J. W. Lee, A. S. Hall, J.-D. Kim and T. E. Mallouk, Chem. Mater., 2012, 24, 1158-1164.

57 H.-W. Wang, Z.-A. Hu, Y.-Q. Chang, Y.-L. Chen, H.-Y. Wu, Z.-Y. Zhang and Y.-Y. Yang, J. Mater. Chem., 2011, 21, 10504.

58 X. Zheng, X. Yan, Y. Sun, Z. Bai, G. Zhang, Y. Shen, Q. Liang and Y. Zhang, ACS Appl. Mater. Interfaces, 2015, 7, 24802485.

59 D. J. Xue, S. Xin, Y. Yan, K. C. Jiang, Y. X. Yin, Y. G. Guo and L. J. Wan, J. Am. Chem. Soc., 2012, 134, 2512-2515.

60 Z. Li, Z. Pan and S. Dai, J. Colloid Interface Sci., 2004, 277, 3542.

61 D.-H. Kim and K. Waki, J. Nanosci. Nanotechnol., 2010, 10, 2375-2380.

62 K. H. Lee, J. Oh, J. G. Son, H. Kim and S. S. Lee, ACS Appl. Mater. Interfaces, 2014, 6, 6361-6368.

63 B. Zheng, T.-W. Chen, F.-N. Xiao, W.-J. Bao and X.-H. Xia, J. Solid State Electrochem., 2013, 17, 1809-1814.

64 P. Wen, P. Gong, Y. Mi, J. Wang and S. Yang, RSC Adv., 2014, 4, 35914.

65 J. Li, Z. Ren, Y. Ren, L. Zhao, S. Wang and J. Yu, RSC Adv., 2014, 4, 35789.

66 Y. Lv, L. Gan, M. Liu, W. Xiong, Z. Xu, D. Zhu and D. S. Wright, J. Power Sources, 2012, 209, 152-157.

67 B. Qiu, C. Pan, W. Qian, Y. Peng, L. Qiu and F. Yan, J. Mater. Chem. A, 2013, 1, 6373.

68 H. Zhao, L. Wang, D. Jia, W. Xia, J. Li and Z. Guo, J. Mater. Chem. A, 2014, 2, 9338.

69 T.-X. Shang, M.-Y. Zhang and X.-J. Jin, RSC Adv., 2014, 4, 39037.

70 H. Yi, H. Wang, Y. Jing, T. Peng and X. Wang, J. Power Sources, 2015, 285, 281-290.

71 C. Tang, Z. Tang and H. Gong, J. Electrochem. Soc., 2012, 159, A651.

72 H. Wang, C. M. B. Holt, Z. Li, X. Tan, B. S. Amirkhiz, Z. Xu, B. C. Olsen, T. Stephenson and D. Mitlin, Nano Res., 2012, 5, 605-617.

73 D.-W. Wang, F. Li and H.-M. Cheng, J. Power Sources, 2008, 185, 1563-1568.

74 C. S. Dai, P. Y. Chien, J. Y. Lin, S. W. Chou, W. K. Wu, P. H. Li, K. Y. Wu and T. W. Lin, ACS Appl. Mater. Interfaces, 2013, 5, 12168-12174. 